\title{
A Cross-sectional Study on Hepatoprotective agents and Antimicrobials in Treatment of Liver Diseases
}

\author{
Allu Haritha ${ }^{1, *}$, Kakaraparthy Ravi shankar ${ }^{2}$, Kondepudi Sai Susmitha ${ }^{3}$, Kaparapu Reshma ${ }^{3}$, \\ Dwarapureddy Chandramouli3 ${ }^{3}$, Manokaran $\mathrm{KM}^{3}$
}

1'Doctor of Pharmacy, Aditya College of Pharmacy Surampalem, Andhra Pradesh, INDIA.

2Department of Pharmacology, Aditya College of Pharmacy, Surampalem, Andhra Pradesh, INDIA.

${ }^{3}$ Doctor of Pharmacy, Aditya College of Pharmacy Surampalem, Andhra Pradesh, INDIA

\begin{abstract}
Background: Liver diseases are one of the global health care problems with enormous social, economic, and clinical consequences accounting for death. Aim: our main aim is to promote the symptoms, complications of liver disease and to reduce the risk of disease, and evaluate the utilization and effectiveness of hepatoprotective agents along with the utilization of antimicrobials. Materials and Methods: This is a 6-month crosssectional study in a tertiary care teaching hospital with a sample size of 55 patients. The patient profile, questionnaire, and laboratory tests were used to gather information. Results: Among 55 patient's alcoholic liver disease (36.36\%) followed by chronic liver disease (34.54\%), decompensated liver disease (12.72\%), and liver abscess (16.36\%) seen at a common age group of $40-70$ years in which males $(87.27 \%)$ and females $(12.72 \%)$ are affected. About $85.45 \%$ are habituated to be alcoholic and $14.54 \%$ are non-alcoholic. About $60 \%$ of patients experiencing ascites as a complication. Mostly prescribed drugs are antibiotics $(80 \%)$ and hepatoprotective agents $(78.18 \%)$ are cephalosporins and ursodeoxycholic acid respectively. This study showed a drop of $42 \%$ Serum bilirubin, 58\% Aspartate transaminase, and 65\% Alanine transaminase in patients having hepatoprotective agents in a time gap of 1 week. Conclusion: The study suggests that abstinence of alcohol along with the management of underlying symptoms with antibiotics and hepatoprotective agents may help in preventing further complications.
\end{abstract}

Key words: Alcoholic liver disease, Decompensated liver disease, Chronic liver disease, Liver abscess, Hepatoprotective agents, Antibiotics.

\section{INTRODUCTION}

Hepatic disease is another term for liver disease. Liver disease is defined as a disruption in liver function that results in sickness. Jaundice, abdominal pain, and swelling, edema, itchy skin, dark urine color, pale or bloody or tarcolored stool, chronic fatigue, nausea or vomiting, anorexia, tendency to bruise easily are common clinical signs of liver disease. ${ }^{1}$ The purpose of liver disease testing is to screen for and identify liver injury as well as to determine the degree of the injury, diagnose the cause and track the condition of the liver over time. Complete Blood Count (CBC), Liver testing, and Liver biopsy are laboratory testing. Ultrasound, computed tomography, magnetic resonance imaging, and magnetic resonance cholangiopancreatography are examples of non-laboratory testing. ${ }^{2}$ Common drugs in liver diseases include Hepatoprotective agents which are used to treat liver damage e.g., Hepamerz, ursodeoxycholic acid, silymarin. Because of suppressing immunity in liver diseases patients are more prone to infections, so antibiotics are used to treat bacterial infections. ${ }^{3}$
DOI: 10.5530/ijopp.14.4.51

Address for correspondence: Dr. Haritha Allu,

Assistant Professor, Doctor of Pharmacy, Aditya College of Pharmacy, Surampalem -533437, Andhra Pradesh, INDIA.

Phone no: +919493493808 Email id: harithaajay14@ gmail.com

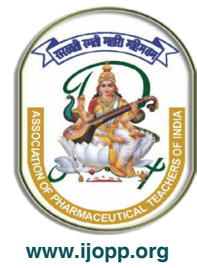


The impairments to the liver and its functioning caused by excessive alcohol intake are defined as Alcoholic liver disease (ALD). Chronic consumption of alcohol intake over a particular daily quantity varies greatly across individuals and can develop the illness. ${ }^{4}$ The three most widely recognized forms of ALD are alcoholic fatty liver (steatosis), acute alcoholic hepatitis, and alcoholic cirrhosis. $^{5}$

Steatosis (alcoholic fatty liver) is a disease that can range from mild liver inflammation to acute liver failure. ${ }^{6}$ Alcoholic hepatitis is a jaundice and liver failure clinical condition that develops after drinking heavily over a lengthy period (mean intake, approximately $100 \mathrm{~g}$ per day). ${ }^{7}$ Cirrhosis follows different mechanisms of liver injury that cause necroinflammation and fibrogenesis; ${ }^{8}$ Cirrhosis is divided into compensated and decompensated phases, each with its own set of characteristics, prognoses, and mortality predictions. Within the compensated stage, two subpopulations have been identified, each with a different prognosis, based on the presence or lack of varices. ${ }^{9}$ The development of consequences of portal hypertension (ascites, variceal hemorrhage, hepatic encephalopathy) or liver insufficiency is classified as decompensated cirrhosis (jaundice). ${ }^{10}$ Right hypochondriac, epigastric, or left upper quadrant stomach discomfort are all symptoms of a liver abscess. ${ }^{11}$

\section{Complications}

Complications of alcoholic liver disease include Oesophageal variceal bleed are dilated oesophageal veins secondary to portal hypertension. When fluid accumulates in the abdomen, between two membrane layers that together make up the peritoneum develops ascites. ${ }^{12}$ An ascitic fluid infection without an obvious intra-abdominal medically curable source develops in patients with cirrhosis and ascites is characterized as spontaneous bacterial peritonitis (SBP). ${ }^{13}$

Hepatorenal syndrome (HRS) is a condition in which people with severe liver disease suffer renal failure. Hepatic encephalopathy, also known as portosystemic encephalopathy (PSE), is a reversible brain dysfunction disease that occurs in people with extensive liver failure. Hepatocellular carcinoma (HCC) is a kind of liver cancer that most commonly affects people who have cirrhosis or chronic liver disease. The term "portal hypertension" refers to an increase in pressure in the portal venous system. The usual value for portal venous pressure is $10 \mathrm{mmHg}$. Patients usually present with splenomegaly, life-threatening gastrointestinal bleeding, and ascites. ${ }^{14}$
Alcoholic abstinence is the most essential aspect of managing alcoholic liver damage. Medications such as naltrexone, acamprosate, and disulfiram are used to treat alcoholism. Vitamins and trace minerals deficiencies $g$ (e.g., Vitamin A, Vitamin D, thiamine, folate, pyridoxine, and zinc) while maintaining a daily intake of $1.2-1.5 \mathrm{~g}$ of protein $/ \mathrm{kg}$ and $35-40 \mathrm{kcal} / \mathrm{kg}$ is suggested to improve nitrogen balance. The agents that prevent hepatic damage are hepatoprotective agents. Ursodeoxycholic acid does not affect long-term survival but reduces blood bilirubin by $25 \%$, serum alanine transaminase by $35 \%$, and aspartate transaminase by $33 \%$. Hence, it is used as an off-label treatment for treating alcoholic liver disease. ${ }^{15}$ Hepamerz is an amino acid mixture of ornithine and aspartate that is used to treat hepatic encephalopathy. ${ }^{16}$ Because liver disease patient's immune is suppressed, they are more susceptible to infections, thus antibiotics are used to treat bacterial infections. Antibiotics should be administered with caution since they can induce acute liver failure.

\section{MATERIALS AND METHODS}

A Cross-sectional study was performed in the Department of General Medicine, GSL General Hospital, Rajahmundry between September 2019 and February 2020. Over six months, 55 patients were monitored. Before being included in the study, each participant was given oral information and a written informed consent form. Patients' case sheets, as well as questionnaires from patients or patient representatives about their alcohol intake, previous health problems, and co-morbidities, were used to collect data. Age, gender, illness prevalence, and medication treatment care offered to patients, particularly antibiotic and hepatoprotective agent use, have all been deemed significant for this research. The information gathered was organized into spreadsheets and evaluated for evaluation and interpretation.

Inclusion Criteria: This research includes patients of both sexes who have been diagnosed with liver disease and are above the age of 20 .

Exclusion Criteria: Patients under the age of 20 and those who refuse to comply are excluded from the study.

\section{Statistical Analysis}

Numbers and percentages are used to represent categorical data. Microsoft Excel was used to produce the data analysis and graphics. To demonstrate the efficiency of hepatoprotective drugs, the collected data were examined using the statistical techniques mean and 
paired $t$-test, and the findings were presented.

\section{RESULTS}

During the six-month research period, a total of 55 individuals were evaluated. The findings reveal that

\section{Disease wise distribution}

The majority of the patients in this study are males $(87.27 \%)$ and females $(12.73 \%)$ alcoholic liver disease $(36.36 \%, n=20)$, chronic liver disease $(34.54 \%, n=19)$, decompensated liver disease $(12.72 \%, n=7)$, liver abscess $(16.36 \%, n=9)$.

\section{Gender wise distribution}

This study includes both sexes in which the majority of the patient are males (87.27\%) and females (12.73\%).

\section{Age-wise distribution}

Patients aged 21-30 $(n=5), 31-40(n=13)$, and over 40 $(n=37)$ had the highest prevalence of liver disease, with the bulk of cases occurring between the ages of 40 and 80 .

\section{Habit wise distribution}

Alcoholic patients are more prone to liver disease than non-alcoholics. In our study alcoholic percentage is $85.45 \%$ and the non-alcoholics percentage is $14.54 \%$.

\section{Symptoms wise distribution}

The most commonly observed symptom among liver diseases are jaundice $(65.45 \%)$, ascites $(60 \%)$, edema (52.72\%), fever and shivering (45.45\%), altered sleep pattern (45.45\%), breathlessness (32.72\%), GI symptoms (26.46\%), early bruising/ bleeding (5.45\%), confusion (3.63\%), clubbing (3.63\%).

\section{Complications wise distribution}

The most commonly observed complications in liver diseases were ascites $(60 \%)$, followed by portal hypertension $(25.45 \%)$, splenomegaly $(23.63 \%)$, hepatomegaly (20\%), hepatic encephalopathy (16.36\%), bleeding disorders (12.72\%), oesophageal varices $(10.90 \%)$, hepatorenal syndrome $(5.45 \%)$, bacterial peritonitis (3.63\%), hepatocellular carcinoma ( $0 \%)$.

\section{Drug wise distribution}

The most prescribed drugs in liver diseases are antibiotics (80\%), hepatoprotective agents $(78.18 \%)$, proton pump inhibitors $(72.72 \%)$, beta blockers $(56.36 \%)$, vitamins
(50.90\%), analgesics $(45.45 \%)$, anti-hemorrhagic (38.18\%), 25\% dextrose (27.27\%), antiemetics $(20 \%)$, minerals $(18.18 \%)$, oral glucose $(16.36 \%)$, vasoactive agents $(12.72 \%)$ and represented in (Figure 1).

\section{Utilization of hepatoprotective agents}

Among hepatoprotective agents the utilization of silymarin $(1.81 \%, n=1)$, 1-ornithine, -1 - aspartate $(72.72 \%$, $\mathrm{n}=40)$, ursodeoxycholic acid $(76.36 \%, \mathrm{n}=42)$ depicted in Table 1.

\section{Effects of hepatoprotectives on serum bilirubin, Aspartate Transaminase (AST), and Alanine Transaminase (ALT)}

The study showed a drop of $42 \%$ of serum bilirubin and $58 \%$ of aspartate transaminase and $65 \%$ of alanine transaminase in patients having hepatoprotective agents in a time gap of one week. This is shown in Figures 2-4 for serum bilirubin, aspartate transaminase, alanine transaminase and represented in Tables (2-4).

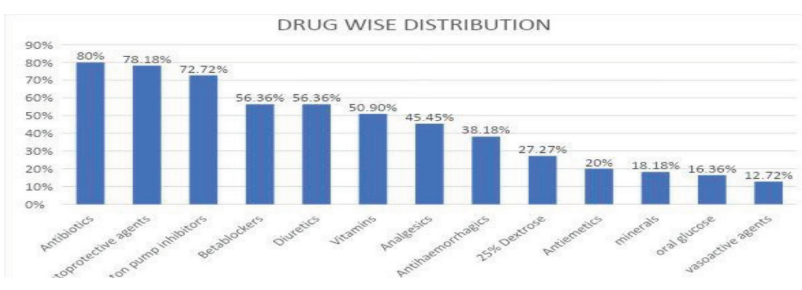

Figure 1: Drug wise distribution.

\begin{tabular}{|c|c|c|c|c|c|}
\hline $\begin{array}{l}\text { Hepatoprotective } \\
\text { agents }\end{array}$ & 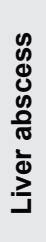 & 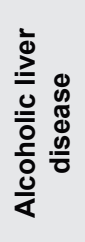 & 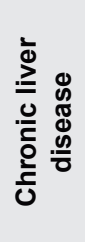 & 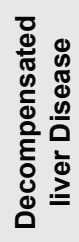 & 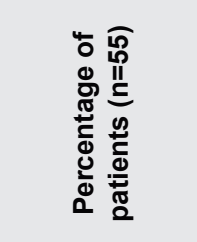 \\
\hline Silymarin & 0 & 1 & 0 & 0 & $1.81 \%(n=1)$ \\
\hline $\begin{array}{l}\text { L-Ornithine, } \\
\text { L-aspartate, } \\
\text { L-pancreatin }\end{array}$ & 3 & 16 & 15 & 6 & $72.72 \%(n=40)$ \\
\hline $\begin{array}{l}\text { ursodeoxycholic } \\
\text { acid }\end{array}$ & 2 & 20 & 14 & 6 & $76.36 \% \quad(n=42)$ \\
\hline
\end{tabular}

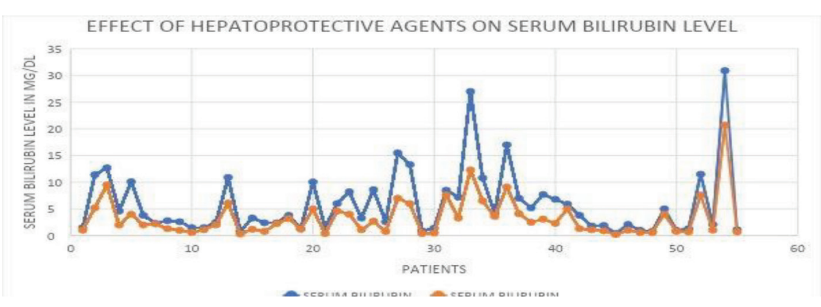

Figure 2: Effect of hepatoprotective agents on serum bilirubin level. 
Table 2: Analysis of serum bilirubin values before and after administration of hepatoprotective agents.

\begin{tabular}{cc} 
Table Analyzed & Paired $\boldsymbol{t}$ test data \\
\hline Column B & After \\
Vs & Vs \\
Column A & Before \\
Paired $t$ test & \\
$P$ value & $<0.05$ \\
Was the pairing significantly & Yes \\
effective? & \\
\hline
\end{tabular}

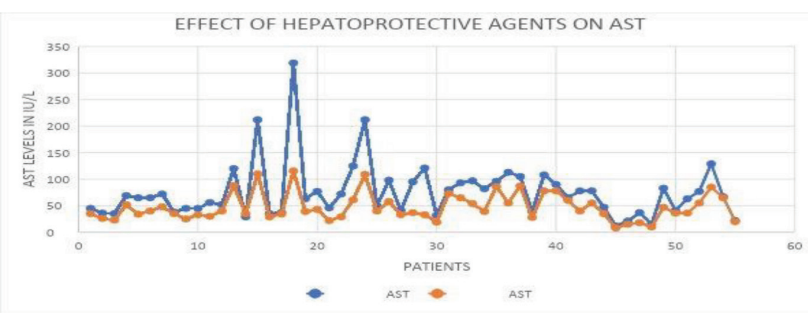

Figure 3: Effect of hepatoprotective agents on aspartate transaminase.

\begin{tabular}{l}
$\begin{array}{l}\text { Table 3: Analysis of aspartate transaminase values } \\
\text { before and after administration of hepatoprotective } \\
\text { agents. }\end{array}$ \\
\begin{tabular}{cc} 
Table Analysed & Paired $t$ test data \\
\hline Column B & After \\
Vs & Vs \\
Column A & Before \\
Paired $t$ test & \\
$P$ value & $<0.05$ \\
Was the pairing significantly \\
effective?
\end{tabular} \\
\hline
\end{tabular}

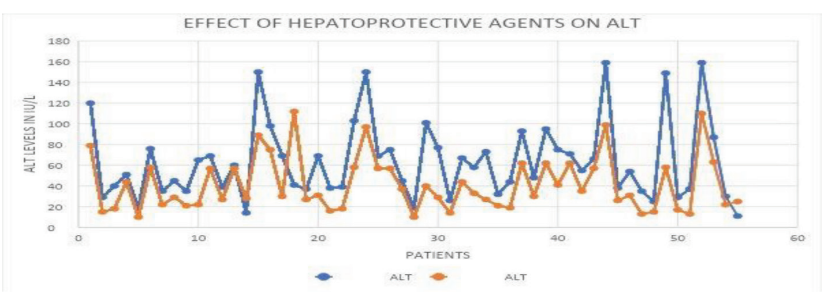

Figure 4: Effect of hepatoprotective agents on alanine transaminase.

\section{DISCUSSION}

The goal of the study was to assess the problems associated with liver illnesses, as well as the use of antibiotics and hepatoprotectives, and the efficacy of hepatoprotective drugs among liver disease patients in the department of general medicine at the GSL hospital in Rajahmundry. With a sample size of 55, the study
Table 4: Analysis of alanine transaminase values before and after administration of hepatoprotective agents.

\begin{tabular}{cc} 
Table Analyzed & Paired $\boldsymbol{t}$ test data \\
\hline Column B & After \\
Vs & Vs \\
Column A & Before \\
Paired $t$ test & \\
$P$ value & $<0.05$ \\
Was the pairing significantly \\
effective?
\end{tabular}

is a cross-sectional study employing patient case sheets and questionnaires on alcoholic intake, health status, and comorbidities.

According to the findings, the most prevalent liver disease $(36.36 \%)$ is alcoholic liver disease, which is caused mostly by excessive alcohol use and is the leading cause of high mortality and morbidity. Chronic liver disease (34.54\%), decompensated liver disease (12.72\%), and liver abscess $(16.36 \%)$.

Even though many studies indicate women develop liver disease by consuming a lower amount of alcohol and for a shorter period compared to men. But in our study, we observed only $12.72 \%$ females and $87.27 \%$ males. This shows that males are more involved in alcoholic intake than females. We also observed people who are habituated to be alcoholic (85\%) and non-alcoholics $(15 \%)$ such as people who experience parasitic infections and hepatitis viruses that infect the liver; fatty liver is more prone to liver diseases. It also showed that most patients experiencing liver diseases are in the age group between $40-70$ years. This can be explained by observing the previous history of the patient's habit of consuming alcohol from a very early age that is from 10 to 15 years.

Christena James et al. conducted prospective research on an assessment of clinical profile in alcoholic liver disease and alcoholic hepatitis. Patients with alcoholic liver disease had a common symptom of stomach discomfort and jaundice, according to their findings. ${ }^{17}$ Similarly, the most frequently observed symptoms in our study were jaundice, ascites, oedema, fever and shivering, altered sleep patterns, breathlessness, and GI symptoms, which included abdominal distension, abdominal pain, blood in vomit or stools, poor appetite, nausea and vomiting, constipation, and diarrhea. Clubbing and confusion are two of the least frequent symptoms.

Ascites (60\%), portal hypertension (25.45\%), 
splenomegaly $(23.63 \%)$, hepatomegaly $(20 \%)$, hepatic encephalopathy (16.36\%), bleeding disorders (12.72\%), oesophageal varices $(10.90 \%)$, hepatorenal syndrome (5.45\%), bacterial peritonitis (3.63\%) and least observed in hepatocellular carcinoma $(0 \%)$ are the most commonly observed complications in our study.

Kolasani, et al. published a retrospective observational research on prescribing patterns in alcoholic liver disease. Antibiotics and hepatoprotectives are the most often recommended drugs, according to the study's findings, followed by symptomatic relief with propranolol, diuretics, intravenous fluids, analgesics, multivitamins, and alcohol withdrawal therapies. ${ }^{18}$ Antibiotics were also the most often prescribed medications in our study (80\%), with Ceftriaxone, ciprofloxacin, metronidazole, sulbactam + cefoperazone, rifaximin, piperacillin + Tazobactam, and levofloxacin being the most usually given drugs in patients based on their condition. Hepatoprotective Agents (78.18 \%) are the second most often recommended medicines, with ursodeoxycholic acid (76.36\%), l-ornithine-l-aspartate (72.72\%), and silymarin being the most widely used $(1.81 \%)$. Acid reflux can be treated with the proton pump inhibitor pantoprazole. To treat portal hypertension, beta-blockers like propranolol are utilized. Oedema and ascites are treated with diuretics such as spironolactone or furosemide.

Vitamins and minerals, such as multivitamins, thiamine, folic acid, and sodium chloride, are recommended since nutritional insufficiency is frequent among these individuals. Pain is relieved by analgesics such as paracetamol. Vitamin $\mathrm{K}$ and tranexamic acid are used as anti-hemorrhagic. The most often administered intravenous fluid is 25 percent dextrose. Nausea and vomiting are treated with anti-emetics such as ondansetron. The use of vasoactive drugs such octreotide, terlipressin, and norepinephrine is recommended.

Hanan Fathima Ahmed studied the effects of hepatoprotective agents in alcoholic liver disease in prospective observational research. In one-week, ursodeoxycholic acid reduces serum bilirubin by $25 \%$, aspartate transaminase by 33\%, and alanine transaminase by $35 \%$. In similar research, individuals receiving hepatoprotective medications had a $42 \%$ reduction in serum bilirubin, a $58 \%$ drop in aspartate transaminase, and a $65 \%$ drop in alanine transaminase in a one week.

\section{Limitation}

Although our study yields important results, it has limitations. It is a study institution of a relatively uncommon patient group with a smaller number of samples. So, a large group and multi-center study would be valuable to evaluate mortality and quality of life.

\section{CONCLUSION}

The current study shows that liver diseases are more prevalent in individuals who have been drinking alcohol for a long time, as well as in patients who have parasite infection, hepatitis viruses that infect the liver, and alcoholic fatty liver. The most prevalent type of liver disease is alcoholic liver disease. Males are more susceptible to liver problems than females, according to the findings, which might be attributed to men's greater alcohol use. The majority of the participants are between the ages of 40 and 80 . Since most patients have comparable symptoms including jaundice, ascites, oedema, fever, and shivering, and discomfort in the abdomen and abdominal distension are common GI symptoms. It has been shown that the length of alcohol intake has a significant impact on the development of problems such as ascites, which is the most frequent complication, and hepatic cancer, which is the least common complication. The best treatment for liver disease starts with therapeutic lifestyle modifications like abstinence from alcohol, which has been shown to improve survival at all stages of the illness. The patient's care is entirely focused on the symptoms and severity of the condition, as well as alcohol abstinence and nutritional assistance. Cephalosporins are the most commonly prescribed antibiotics. Hepatoprotectives are also used to enhance the liver's function, which is affected by liver disease. A reduction of $42 \%$ in serum bilirubin, $58 \%$ in aspartate transaminase, and $65 \%$ in alanine transaminase were observed in patients receiving hepatoprotective medications during one week, according to the research.

\section{ACKNOWLEDGEMENT}

The authors would like to thank Dr. M. Sri Haribabu, General Medicine, (GSL medical college and hospital) for his prompt guidance and scientific approach, as well as thank everyone else who contributed to the thesis's success.

\section{CONFLICT OF INTEREST}

The authors declare that there is no conflict of interest. 


\section{ABBREVIATION}

ALD: Alcoholic Liver Disease; ALP: Alkaline Phosphotase; ALT: Alanine Amino Transferase; ASH: Alcoholic Steatohepatitis; CBC: Complete Blood Count; CLD: Chronic Liver Disease; CT: Computerized Tomography; DLD: Decompensated Liver Disease; FDA: Food and Drug Adminstration; GI: Gastro Intestine; HCC: Hepato Cellular Carcinoma; HCV: Hepatitis C Virus; HE: Hepatic Encephalopathy; HRS: Hepatic Renal Syndrome; SBP: Spontaneous Bacterial peritonitis; SGOT: Serum Glutamic Oxaloacetic Transaminase; SGPT: Serum Glutamic Pyruvate Transaminase.

\section{SUMMARY}

The goal of the study was to determine the consequences of alcoholic liver disease, as well as the use of antimicrobials and hepatoprotective agents, as well as the efficacy of hepatoprotective drugs in the treatment of liver disorders. 55 patients of both sexes attending a tertiary care center were screened and analyzed, and we found that ascites as the most common complication. We also noticed that cephalosporins are the most commonly used antibiotic and that using hepatoprotective agents for a week results in a decrease in serum bilirubin, aspartate transaminase, and alanine transaminase.

\section{REFERENCES}

1. Wedro Benjamin, Liver Disease Symptoms, Signs, Diet and Treatment Medicine Net https://www.medicinenet.com > liver disease > article9.

2. Liver disease-diagnosis. Available from: https://labtestsonline.org/conditions/ liver-disease [cited 24/7/2021].

3. Amarapurkar Deepak N. Prescribing medications in patients with decompensated liver cirrhosis. Int J Hepatol. 2011;2011:519526. doi: 10.4061/2011/519526, PMID 21994861.
4. Kong Ling-Zu, Chandimali Nisansala, Han Ying-Hao, Lee Dong-Ho, Kim Ji-Su, Kim Sun-Uk, Kim Tae-Don, Jeong Dong Kee, Sun Hu-Nan, Lee Dong Sun, Kwon Taeho. Pathogenesis, early diagnosis, and therapeutic management of alcoholic liver disease. Int J Mol Sci. 2019;20(11):2712. doi: 10.3390/ ijms20112712, PMID 31159489.

5. Walsh K, Alexander GKevin Walsh, et al.. alcoholic liver disease. Postgrad Med J. 2000;76(895):280-6. doi: 10.1136/pmj.76.895.280, PMID 10775280.

6. Tilg Herbert, Day Christopher P. Management strategies in alcoholic liver disease. Nat Clin Pract Gastroenterol Hepatol. January 2007;4(1):24-34. doi: 10.1038/ncpgasthep0683, PMID 17203086.

7. Jackson Phil, et al. Alcoholic liver disease, Continuing Education in Anesthesia, Critical Care and Pain j. Vol. 10(3); 2010.

8. Tsochatzis Emmanuel A, Bosch J, Burroughs AK. Liver cirrhosis. The Lancet. 2014;383(9930):1749-61. doi: 10.1016/S0140-6736(14)60121-5.

9. Garcia-Tsao Guadalupe, Friedman Scott, Iredale John, Pinzani Massimo. Now there are many (stages) where before there was one: in search of a pathophysiological classification of cirrhosis. Hepatology. 2010;51(4):1445-9. doi: 10.1002/hep.23478, PMID 20077563.

10. Oesophageal variceal bleed - Oxford Medical Education. Available from: http:// www.oxfordmedicaleducation.com/gastroenterology/oesophageal-varicealbleed [cited 24/7/2021].

11. Jayakar Sudhir R, et al. Liver abscess, management strategies, and outcome, International Surgery. Journal | September. 2018 | Vol 5 | Issue 9.

12. Decompensated chronic liver disease, oxford medical condition. Available from: http://www.oxfordmedicaleducation.com/wp-content/uploads/2014/10/ Chronic-liver-disease-Decompensated-N.pdf [cited 24/7/2021].

13. Bruce A rayon, et al. Spontaneous bacterial peritonitis in adults: diagnosis https://www.uptodate.com/contents/spontaneous-bacterial-peritonitis-inadults-diagnosis/contributors.

14. Brig J.K. Banerjee, Portal hypertension. Med J Armed Forces India 68. 2012:276-9.

15. Ahmed Hanan Fathima, Naseem S, Unnisa H, Amreen A, Ansari JA, Sultana R. A prospective observational study on effects of hepatoprotective agents in alcoholic liver disease at a tertiary care hospital. J Drug Delivery Ther. 2018;8(1):7-12. doi: 10.22270/jddt.v8i1.1544.

16. Butterworth Roger F, Canbay Ali Hepatoprotection by L-Ornithine L-Aspartate in Non-Alcoholic Fatty Liver Disease. Dig Dis. 2019;37(1):63-8. doi: 10.1159/000491429. PMID 30016770.

17. James Christeena, et al. Assessment of clinical profile and prescription pattern of drugs in alcoholic liver disease and hepatitis in a tertiary care hospital. Int J Pharm Sci Rev Res;46(1), September-october2017:Article Number 07.

18. Kolasani Bhanu Prakash, Sasidharan P, Cm D, Jayabal P, Rajaseharan A. Prescribing pattern of drugs in patients with alcoholic liver disease in tertiary care teaching hospital. Natl J Physiol Pharm Pharmacol. january 27 2017;7(5). doi: 10.5455/njppp.2017.7.1233027012017. 